1 Biosynthetic gene cluster profiling predicts the positive association between antagonism and

2

3

4

\title{
phylogeny in Bacillus
}

\section{Authors:}

Liming Xia ${ }^{1 \S}$, Youzhi Miao ${ }^{1 \S}$, A $^{\prime} l i \mathrm{Cao}^{1}$, Yan Liu ${ }^{1}$, Zihao Liu ${ }^{1}$, Xinli Sun ${ }^{1}$, Yansheng Xue ${ }^{1}$, Zhihui $\mathrm{Xu}^{1}$, Weibing Xun ${ }^{1}$, Qirong Shen ${ }^{1}$, Nan Zhang ${ }^{1^{*}}$, Ruifu Zhang ${ }^{2 *}$

\section{Affiliations:}

${ }^{1}$ Jiangsu Provincial Key Lab of Solid Organic Waste Utilization, Jiangsu Collaborative Innovation Center of Solid Organic Wastes, Educational Ministry Engineering Center of Resource-saving fertilizers, Nanjing Agricultural University, Nanjing 210095, Jiangsu, P. R. China

${ }^{2}$ Key Laboratory of Microbial Resources Collection and Preservation, Ministry of Agriculture, Institute of Agricultural Resources and Regional Planning, Chinese Academy of Agricultural Sciences, Beijing 100081, P. R. China

${ }^{\S}$ Both authors contributed equally to this paper.

\section{${ }^{*}$ Corresponding authors:}

Nan Zhang, College of Resources \& Environmental Sciences, Nanjing Agricultural University, 210095, Nanjing, P. R. China E-mail: nanzhang@njau.edu.cn.

Ruifu Zhang, Institute of Agricultural Resources and Regional Planning, Chinese Academy of Agricultural Sciences, Beijing 100081, P. R. China; E-mail: zhangruifu@caas.cn.

The authors declare no conflict of interest. 
Abstract: Understanding the driving forces and intrinsic mechanisms of microbial competition is a fundamental question in microbial ecology. Despite the well-established negative correlation between exploitation competition and phylogenetic distance, the process of interference competition that is exemplified by antagonism remains controversial. Here, we studied the genus Bacillus, a commonly recognized producer of multifarious antibiotics, to explore the role of phylogenetic patterns of biosynthetic gene clusters (BGCs) in mediating the relationship between antagonism and phylogeny. Comparative genomic analysis revealed a positive association between BGC distance and phylogenetic distance. Antagonistic tests demonstrated that the inhibition phenotype positively correlated with both phylogenetic and predicted BGC distance, especially for antagonistic strains possessing abundant BGCs. Mutant-based verification showed that the antagonism was dependent on the BGCs that specifically harbored by the antagonistic strain. These findings highlight that BGC-phylogeny coherence regulates the positive correlation between congeneric antagonism and phylogenetic distance, which deepens our understanding of the driving force and intrinsic mechanism of microbial interactions.

Keywords: antagonism; biosynthetic gene clusters (BGCs); phylogenetic distance; Bacillus

\section{Introduction}

Microbes are naturally surrounded by taxonomically different ones with which they compete for scarce resources and space ${ }^{1}$. Competition between different species is generally categorized as exploitation competition, which involves the rapid consumption of a limited resource ${ }^{2,3}$, and interference competition, which refers to direct antagonistic interactions ${ }^{4}$. Microbial antagonism is 
driven by diverse toxins, such as broad-spectrum antibiotics and strain-specific bacteriocins ${ }^{5,6}$, and is recognized as a key element regulating populations and determining their success within diverse communities ${ }^{7}$. The stunning diversity of both the categories and functions of antimicrobial metabolites among different species, results in the extraordinary complexity of interference competition between microbes with different phylogenetic relationships ${ }^{4,8}$. Accordingly, illustrating the driving forces and mechanisms of antagonistic competition is crucial for understanding and predicting microbial behaviors during community assemblage and succession ${ }^{2}$.

Phylogenetic relatedness is considered to be closely associated with microbial competition, based on its determination of both primary and secondary metabolic profiles ${ }^{2,4}$. Despite the wellestablished negative correlation between exploitation competition and phylogenetic distance ${ }^{2}$, the process of interference competition is much more complicated and remains controversial. Russel et al. demonstrated that inhibition was more prevalent between closely related bacteria, and this negative correlation between antagonism and phylogeny was mediated by the overlap of the metabolic niche among different strains ${ }^{9}$. Conversely, other studies examined congeneric competition in Vibrio and Streptomyces and revealed that closely related strains competed less than phylogenetically distant strains, which was probably caused by the effect of the prior coexistence and distribution of secondary metabolites in different genomes ${ }^{7,10,11}$. Additionally, the positive relationship between kin discrimination and phylogeny was indicated within Bacillus subtilis, which was modulated by genes involved in antimicrobials and cell-surface modifiers ${ }^{12,13}$; however, this correlation was lost or even became a certain extent of negative when more distantly Bacillus strains were tested for antagonism, probably being dependent on the demand of protecting public goods ${ }^{14}$. 
Taken together, the relationship between antagonism and phylogenetic distance with regard to microbes from different taxonomical scale or groups, as well as the involved biological mechanism, is still under debate, which limits both our understanding and application of these microbial interactions.

Biosynthetic gene clusters (BGCs) are responsible for the production of various secondary metabolites that contribute to interference competition between different microbes ${ }^{15,16}$, and also usually provide resistance against the self-produced antibiotic to protect the host cell ${ }^{4,17,18}$. Although the relevance (or lack of relevance) of BGCs to antagonism or phylogeny have been evaluated in diverse microbes ${ }^{11,16,17,19-21}$, the involvement of BGCs profile in mediating the relationship between interference competition and phylogeny, has not been well addressed. Here, we hypothesize that the correlation between BGC and phylogenetic distance can predict the pattern of congeneric antagonism among different taxonomic groups, as strains possessing higher BGC similarity should have a lower probability of inhibiting each other. To test this hypothesis, we referred to the Grampositive Bacillus as the target genus, which is a commonly recognized producer of multifarious secondary metabolites ${ }^{16,19,22-25}$, including non-ribosomal lipopeptides (e.g., surfactin, iturin, and fengycin families produced by various species) ${ }^{23}$, non-ribosomal polyketides (e.g., difficidin and macrolactin produced by $B$. amyloliquefaciens and $B$. velezensis $)^{19}$, peptide-polyketide hybrid compound (e.g., zwittermicin produced by B. cereus and B. thuringiensis) ${ }^{24}$, and ribosomally synthesized and post-translationally modified peptides (RiPPs; e.g., lichenicidin produced by $B$. licheniformis $)^{26}$. We attempted to address the role of the BGC-phylogeny relevance in shaping the association between antagonism and phylogenetic distance based on comparative genomic analysis, 
antagonistic assessments, and mutant-based verification. This study provides new insights to better understand the driving force and mechanism of interference competition in microbial ecology, which will allow us to better manipulate community assemblages for practical purpose.

\section{Results}

Positive correlation between biosynthetic gene cluster (BGC) and phylogenetic distance in the genus Bacillus

BGCs are responsible for the synthesis of secondary metabolites involved in microbial interference competition. To investigate the relationship between BGC and phylogenetic distance within the genus Bacillus, we collected 4,268 available Bacillus genomes covering 139 species from the NCBI database (Table S1). Phylogenetic analysis based on the sequences of 120 ubiquitous single-copy proteins $^{27}$ showed that the 139 species could be generally clustered into four clades (Fig. 1 \& Table S2; the phylogenetic tree including all the detailed species information is shown in Fig. S1), including a subtilis clade that includes species from diverse niches and can be further divided into the subtilis and pumilus subclades, a cereus clade that contains typical pathogenic species (B. cereus, $B$. anthracis, B. thuringiensis, etc.), a megaterium clade, and a circulans clade.

Prediction using the bioinformatic tool antiSMASH ${ }^{15}$ detected 49,671 putative BGCs in the 4,268 genomes, corresponding to an average of 11.6 BGCs per genome (Table S3). The subtilis clade had the most BGCs, 13.1 BGCs per genome, followed by the cereus and megaterium clades, while the circulans clade only had 4.3 BGCs/genome (Fig. 2a; Table S4). The two most abundant BGC classes were nonribosomal peptide-synthetase (NRPS) and RiPPs, which had an abundance of 3.7 
and 3.1 per genome on average, respectively (Fig. S2 \& Table S4). Interestingly, subtilis clade accommodated significantly higher abundance of BGCs in other polyketide synthase (PKSother; 2.0 per genome vs. 0.0 1.1 per genome) and PKS-NRPS_Hybrids (0.7 vs. 0.0 0.2) classes, as compared with the three other clades (Table S4); while cereus clade had more BGCs in RiPPs than other clades on average (Table S4). Overall, the profile of BGC products and classification was generally consistent with the phylogenetic tree (Fig. S3).

To further evaluate whether the diversity and concrete distribution of the BGCs among genomes were relevant to the phylogenetic relatedness, we selected 545 representative Bacillus genomes based on the following criteria: (i) high genome sequencing quality for further BGC distance calculation, and (ii) covering all Bacillus species. We analyzed the interactive sequence similarity network of BGCs in these genomes by using the "biosynthetic gene similarity clustering and prospecting engine" (BiG-SCAPE) ${ }^{28}$. The (dis)similarity of paired BGCs was calculated based on a combination of three metrics, including the Jaccard index (JI), adjacency index (Al), and domain sequence similarity (DSS), which resulted in 1,110 gene cluster families (GCFs) and 76 gene cluster clans (GCCs) of the 4,877 putative BGCs (Tables S5 \& S6). The hierarchal clustering based on the abundance of these GCFs among each genome (Table S7) indicated that, each phylogenetic clade/subclade revealed its own distinctive BGCs distribution profile, and possessed a number of taxonomy-specific secondary metabolites (Figs. 2b \& S4; Table S5). The widespread BGCs in cereus clade included fengycin, bacillibactin, bacteriocin, NRPS, and petrobactin, in which petrobactin was a clade-specific BGC; polyoxypeptin, thurincin, and zwittermicin were also specific molecules but were mainly present in a certain of B. cereus and B. thuringiensis genomes (Figs. $2 b$ \& S4). In subtilis 
clade, most species possessed surfactin, fengycin, bacilysin, bacillibactin, and T3PKS, while each surfactin, and T3PKS, some strains can potentially produce lanthipeptide, paeninodin, or bacteriocins. The dominating BGC in circulans clade was identified as T3PKS, and some species may synthesize siderophore, bacteriocin, or lanthipeptide (Figs. 2b \& S4).

GCFs clustering data, and found a significant positive correlation between the BGC and phylogenetic distance (Fig. 2c) $\left(P<0.001, R^{2}=0.2847\right)$. Interestingly, the BGC distance of genomes within or

Fig. S5d) but some can be very remote (e.g., a certain of connections between circulans clade and profile was generally dependent on the phylogenetic relationship within the genus Bacillus.

\section{Antagonism positively correlates with both the phylogenetic and BGC distance in Bacillus}

BGCs not only contribute to the synthesis of secondary metabolites but also usually afford self- 
coherence in Bacillus (Fig. 2b \& 2c) determines a positive correlation between antagonism and

phylogenetic distance. To verify this hypothesis, we first used the bacterial colony confrontation

assay to investigate the relationship between the antagonistic efficiency and phylogenetic distance

of the paired strains (Fig. S6). The antagonistic bacteria included eight strains from the subtilis or

cereus clade, which are the two dominant groups within the genus Bacillus and have been explored species than towards closely related species. For instance, B. amyloliquefaciens ACCC19745 and B. 
This strategy can avoid potential bacterial nutrient competition and is feasible for a wider range of 
can partially explain the weak correlation between antagonism and phylogenetic distance in strains possessing fewer BGCs.

\section{The positive correlation of antagonism and phylogenetic distance in Bacillus is mediated by the} specifically harbored BGCs in antagonistic strains

Having determined that the positive correlation between antagonism and phylogenetic distance was consistent with the BGC-phylogeny coherence in Bacillus, we further investigated the mediation mechanism of BGCs in the interspecies interactions. We used a typical secondary metabolite producer, B. velezensis SQR9 belonging to subtilis clade, to identify the primary antagonistic antibiotic towards different strains. Strain SQR9 devotes approximately $9.9 \%$ of its genome to the synthesis of various antimicrobial metabolites ${ }^{30}$, including five nonribosomal lipopeptides or dipeptides (surfactin, bacillomycin D, fengycin, bacillibactin, and bacilysin), three polyketides (macrolactin, bacillaene, and difficidin) ${ }^{31}$, and one antimicrobial fatty acid (FA; bacillunoic acid) ${ }^{18}$. The antagonistic characteristics of SQR9 mutants deficient in each of the above BGCs and SQR9 $\Delta f p$ with the 4'-phosphopantetheinyl transferase gene deleted and only bacilysin can be synthesized ${ }^{22,30}$ (Table S8), towards 24 target strains (Table S8) were investigated using a fermentation supernatant inhibition assay. SQR9 $\Delta s f p$ nearly completely lost its antagonism towards all the target strains, suggesting that the synthesis of the antibiotics involved in congeneric antagonism is strongly dependent on Sfp (Fig. 5). The active antimicrobial metabolites were found to be relevant to the phylogenetic positions of the target strains, as a specific BGC was involved in the inhibition of strains in one taxonomic group. In detail, difficidin dominated the suppression of the megaterium clade (Fig. 
5); macrolactin was the primary antibiotic against the cereus clade (Fig. 5); difficidin and bacillaene

antimicrobial FA to compete with strains in closely related species (B. halotolerans CF7, B. complicated molecular mechanisms and still needs further investigation.

\section{Discussion}


has been examined in various organisms and has received both positive and negative support ${ }^{33-36}$.

With regard to the microbial fierce competition exemplified by antagonism, Russel et al. found a

negative correlation between inhibition probability and phylogenetic distance ${ }^{9}$; some other

scientists discovered a positive relationship between antagonistic interaction (including kin discrimination) and phylogenetic dissimilarity in genus Vibrio $^{7}$ and Streptomyces ${ }^{10,11}$, and species $B$. subtilis $^{12,13}$. The present study demonstrated that antagonism tended to be positively correlated with phylogenetic distance within the genus Bacillus (Figs. 3 \& 4). Comprehensively, we pronounce interactions (Figs. 2 5). BGCs themselves, or other elements in the same genome, usually afford self-resistance by providing active efflux or modification of the relevant antibiotic ${ }^{29,37}$, while the absence of a specific BGC suggests the potential to be sensitive to this metabolite ${ }^{17,18}$; this principle was also confirmed by the observation that antagonism was dependent on the BGC that was present in the antagonistic strain but absent in the target strain (Fig. 5). In addition, despite the mobility of BGCs among different microbes ${ }^{16,38}$, their distribution pattern was generally in accordance with the phylogenetic relationship within genus (Fig. $2 b$ \& 2c). Consequently, closely related species with a higher BGC similarity have a lower probability of inhibiting each other, while distant species in the same genus are likely to suppress each other more fiercely (Figs. $3 \& 4$ ). Therefore, we highlighted that the coherence between BGCs distribution and phylogenetic characteristics is one of the crucial 
can contribute to an irregular correlation between the secondary metabolite profile and

phylogenetic distance ${ }^{28,39,40}$. In this situation, functional similarity, such as metabolic niche overlap,

may become the main driver and lead to a negative correlation between antagonism and

phylogenetic distance ${ }^{9}$. Taken together, this taxonomic range-dependent association between

antagonism and phylogeny should indicate a cooperation-competition tradeoff during microbial

interactions and is coordinated by a set of sophisticated molecular mechanisms.

Interestingly, the positive correlation between antagonism and phylogeny was relatively strong and phylogeny in these strains. Furthermore, other potential factors may also contribute the 
environmental factors and competing strains) can affect the antibiotic production ${ }^{11,17,41,42}$, and the undiscovered genetic and physiological features may also regulate the response to different predicted BGCs. It would be important to identify more secondary metabolites responsible for bacterial interference competition and to further investigate the exquisite regulation characteristics of these functional molecules.

Noticeably, there are some differences between our finding and that reported by Lyons \&

Kolter, who demonstrated a negative correlation between kin discrimination and phylogeny ${ }^{14}$. This evaluated by colony confrontation and fermentation supernatant inhibition, which was dominated by diffusible secondary antibiotics within a comparatively longer distance; while the kin discrimination in Lyons \& Kolter's study was assessed based on swarm interaction, biofilm meeting, and halo formation ${ }^{14}$, which was likely to be dependent on closer cell association (e.g., toxinantitoxin system and cell surface contact) ${ }^{13}$. (ii) The antagonism-phylogeny correlation in our study was calculated based on interactions between diverse antagonistic and target strains. However, the halo assay performed previously examined the inhibition of one indicated species (B. subtilis NCIB3610) by different testing strains ${ }^{14}$; as discussed above, the different BGCs distribution patterns (e.g., the quantity) among distinct antagonistic strains can influence the inhibition phenotype and

289 its relationship with phylogenetic distance. In general, we consider that Lyons \& Kolter's study has provided important knowledge with regard to kin discrimination and close contact, especially in a which can occur within a longer distance. 
Bacilli possess an amazing capacity to synthesize a diverse range of secondary metabolites;

previous studies have indicated the phylogenetic conservation of BGCs in the genus Bacillus and identified multiple species/clade-specific molecules ${ }^{16,17,43}$. Based on bioinformatics analysis of 
related species can hardly share public goods but still confront nutrient competition; thus, the

competition $^{9}$. As a result, metabolic similarity facilitates the negative correlation between regulating community assemblage and succession ${ }^{47}$. especially in strains possessing abundant BGCs. We expect this positive association between 


\section{Bacterial strains and growth conditions}

All 90 Bacillus strains used in this study are listed in Table S8, including 3 strains obtained from the Bacillus Genetic Stock Center (BGSC), 17 strains obtained from the Agricultural Culture Collection of China (ACCC) that originated from different environmental samples, 60 strains isolated from the plant rhizosphere by this laboratory, and 10 mutants of $B$. velezensis SQR9. All strains were grown at $30^{\circ} \mathrm{C}$ in low-salt Luria-Bertani (LLB) medium (10 $\mathrm{g} \mathrm{L}^{-1}$ peptone; $5 \mathrm{~g} \mathrm{~L}^{-1}$ yeast extract; $\left.3 \mathrm{~g} \mathrm{~L}^{-1} \mathrm{NaCl}\right)$; when necessary, final concentrations of antibiotics were added as follows: $100 \mathrm{mg} \mathrm{L}^{-1}$ spectinomycin (Spc) and $20 \mathrm{mg} \mathrm{L}^{-1}$ zeocin (Zeo). To collect the fermentation supernatant for antagonism assessment, the bacterial strains were cultured in Landy medium ${ }^{48}$. The $16 \mathrm{~S}$ rRNA genes were amplified with the $27 F$ (5'-AGAGTTTGATCCTGGCTCAG-3') and 1492R (5'-GGTTACCTTGTTACGACTT-3') primers and were subsequently Sanger sequenced. The taxonomic affiliations of these strains were determined through the EzBioCloud and NCBI databases.

\section{Bacillus genomic, phylogenetic, and biosynthetic gene cluster analysis}

In total, 4,268 available genomes from 139 different Bacillus species were downloaded from the NCBI database using the ncbi-genome-download script (https://github.com/kblin/ncbigenomedownload/) (Table S1). Then, a phylogenetic tree was constructed based on the concatenation of 120 ubiquitous single-copy proteins using GTDB-Tk 1.4 .1 software with the default parameters ${ }^{27,49}$. The resulting tree was subsequently visualized and edited with Figtree 1.4.4 (http://tree.bio.ed.ac.uk/software/figtree/). Maximum likelihood (ML) phylogenetic trees of the $16 \mathrm{~S}$ rRNA sequence of these strains used in antagonism assessments were constructed by MEGA 5.0. 
profiling, in order to compare the BGC distribution among different Bacillus groups more adequately,

\section{Antagonism assay}

The inhibition of antagonistic strains on target strains (listed in Table S8) was evaluated by both 
379 fermentation supernatant inhibition assay, $150 \mu \mathrm{L}$ of the filtration-sterilized extracellular

\section{B. velezensis SQR9 mutant construction}

Marker-free deletion strains of the target BGC genes were constructed using the strategy previously the transformants were selected via first-step screening on LLB plates containing Spc. The final 


\section{Statistics}

400 LM were performed in the R package (version 3.6.1) to assess the correlation between BGC distance

401

402

403

404

405

406

407

408

409

410 S8.

31972512, and 32072665), the Fundamental Research Funds for the Central Universities

and phylogenetic distance, inhibition phenotype and phylogenetic distance/predicted BGC distance, as well as the association of antagonism-phylogeny/BGC-phylogenetic distance/antagonism-BGC distance and the quantity of BGCs in antagonistic strains. Statistical significance was based on a $P$ value $<0.01$. Graphs of the correlation data were created using the "ggplot2" package in R; the gray shaded areas denote the $95 \%$ confidence intervals. The Duncan's multiple rang tests $(P<0.05)$ of the SPSS version 22.0 (IBM, Chicago, IL, version 22.0) was used for statistical analysis of differences among treatments.

\section{Data availability}

The authors confirm that the data supporting the findings of this study are available within the article or from the corresponding authors upon request. The DNA sequences from all incubation samples are deposited in the NCBI Sequence GenBank database with accession number listed in Supplementary Table

\section{Acknowledgements}

This work was financially supported by the National Natural Science Foundation of China (42090060, (KJQN201919), and the Agricultural Science and Technology Innovation Program of CAAS (CAAS- 
ZDRW202009).

420

421

422

423

424

425

426

427

\section{Author contributions}

Q.S., N.Z. and R.Z. conceived and designed the study. L.X. and A.C. performed experimental work. L.X., Y.M., Y.L., Z.L., Z.X. and W.X. analyzed the data. Y.L., X.S. and Y.X. contributed materials. N.Z., R.Z., Y.M. and L.X. wrote the manuscript.

\section{Conflict of Interest}

The authors declare no conflict of interest.

\section{References}

1. Pérez-Gutiérrez, R.-A. et al. Antagonism influences assembly of a Bacillus guild in a local community and is depicted as a food-chain network. ISME J 7, 487-497 (2013).

2. Ghoul, M. \& Mitri, S. The ecology and evolution of microbial competition. Trends Microbiol 24, 833845 (2016).

3. Hibbing, M.E., Fuqua, C., Parsek, M.R. \& Peterson, S.B. Bacterial competition: surviving and thriving in the microbial jungle. Nat Rev Microbio/ 8, 15-25 (2010).

4. Granato, E.T., Meiller-Legrand, T.A. \& Foster, K.R. The evolution and ecology of bacterial warfare. Curr Biol 29, R521-R537 (2019).

5. Gonzalez, D., Sabnis, A., Foster, K.R. \& Mavridou, D.A.I. Costs and benefits of provocation in bacterial warfare. Proc Natl Acad Sci U S A 115, 7593-7598 (2018).

6. Jamet, A. \& Nassif, X. New players in the toxin field: polymorphic toxin systems in bacteria. mBio 6, e00285-15 (2015).

7. Cordero, O.X. et al. Ecological populations of bacteria act as socially cohesive units of antibiotic 
production and resistance. Science 337, 1228-31 (2012).

8. P., d.V.R. et al. Comparative genomics reveals high biological diversity and specific adaptations in the industrially and medically important fungal genus Aspergillus. Genome Biol 18, 28 (2017).

9. Russel, J., Røder, H.L., Madsen, J.S., Burmølle, M. \& Sørensen, S.J. Antagonism correlates with metabolic similarity in diverse bacteria. Proc Natl Acad Sci U S A 114, 10684-10688 (2017).

10. Vetsigian, K., Jajoo, R. \& Kishony, R. Structure and evolution of Streptomyces interaction networks in soil and in silico. PLoS Biol 9, e1001184 (2011).

11. Westhoff, S., Kloosterman, A., van Hoesel, S.F.A., van Wezel, G.P. \& Rozen, D.E. Competition sensing alters antibiotic production in Streptomyces. mBio 12, e02729-20 (2021). sympatric Bacillus subtilis isolates. PNAS 112, 14042-14047 (2015).

13. Lyons, N.A., Kraigher, B., Stefanic, P., Mandic-Mulec, I. \& Kolter, R. A combinatorial kin discrimination system in Bacillus subtilis. Curr Biol 26, 733-742 (2016). related species. mBio 8, e00723-17 (2017).

15. Blin, K. et al. antiSMASH 5.0: updates to the secondary metabolite genome mining pipeline. Nucleic Acids Res 47, W81-W87 (2019). in the Bacillus subtilis species complex. mSystems 6, e00057-21 (2021). against plant pathogenic fungi. mSystems 6, e00770-20 (2021). 
461 18. Wang, D. et al. A genomic island in a plant beneficial rhizobacterium encodes novel antimicrobial fatty acids and a self-protection shield to enhance its competition. Environ Microbiol (2019).

19. Chowdhury, S.P., Hartmann, A., Gao, X. \& Borriss, R. Biocontrol mechanism by root-associated Bacillus amyloliquefaciens FZB42 - a review. Front Microbiol 6, 780 (2015).

20. Gross, H. \& Loper, J.E. Genomics of secondary metabolite production by Pseudomonas spp. Nat Prod

$\operatorname{Rep} 26,1408-1446$ (2009).

21. Chen, X. et al. Structural and functional characterization of three polyketide synthase gene clusters in Bacillus amyloliquefaciens FZB 42. J Bacteriol 188, 4024-4036 (2006).

22. Chen, X.H. et al. Comparative analysis of the complete genome sequence of the plant growthpromoting bacterium Bacillus amyloliquefaciens FZB42. Nat Biotechnol 25, 1007-14 (2007).

23. Ongena, M. \& Jacques, P. Bacillus lipopeptides: versatile weapons for plant disease biocontrol. Trends Microbiol 16, 115-125 (2008). (2011).

25. Zhao, X. \& Kuipers, O.P. Identification and classification of known and putative antimicrobial compounds produced by a wide variety of Bacillales species. BMC Genomics 17, 882 (2016). translationally modified peptides (RiPPs) in anaerobic bacteria. BMC Genomics 15, 983 (2014). the tree of life. Nat Biotechnol 36, 996-1004 (2018). 
Nat Chem Biol 16, 60-68 (2020).

29. Wilson, D.N. Ribosome-targeting antibiotics and mechanisms of bacterial resistance. Nat Rev Microbiol 12, 35-48 (2014).

30. Zhang, N. et al. Whole transcriptomic analysis of the plant-beneficial rhizobacterium Bacillus amyloliquefaciens SQR9 during enhanced biofilm formation regulated by maize root exudates. BMC Genomics 16, 685 (2015).

31. Xu, Z. et al. Enhanced control of cucumber wilt disease by Bacillus amyloliquefaciens SQR9 by altering the regulation of its DegU phosphorylation. Appl Environ Microbiol 80, 2941-2950 (2014).

32. Darwin, C. On the Origin of Species. John Murray, London (1859).

33. Alexandrou, M.A. et al. Evolutionary relatedness does not predict competition and co-occurrence in natural or experimental communities of green algae. Proc Biol Sci 282, 20141745 (2015).

34. Kunstler, G. et al. Plant functional traits have globally consistent effects on competition. Nature 529, 204-7 (2016).

35. Venail, P.A. et al. The influence of phylogenetic relatedness on species interactions among freshwater green algae in a mesocosm experiment. J Ecol 102, 1288-1299 (2014).

497 36. Violle, C., Nemergut, D.R., Pu, Z. \& Jiang, L. Phylogenetic limiting similarity and competitive exclusion. Ecol Lett 14, 782-7 (2011). Actinobacteria: new concepts for natural product discovery. Nat Rev Microbiol 18, 546-558 (2020). metabolite gene cluster between fungi. Genome Bio/ 9, R18 (2008). 
39. Crits-Christoph, A., Diamond, S., Butterfield, C.N., Thomas, B.C. \& Banfield, J.F. Novel soil bacteria possess diverse genes for secondary metabolite biosynthesis. Nature 558, 440-444 (2018).

40. Dittmann, E., Gugger, M., Sivonen, K. \& Fewer, D.P. Natural product biosynthetic diversity and comparative genomics of the Cyanobacteria. Trends Microbiol 23, 642-652 (2015).

41. Yun, C.S., Motoyama, T. \& Osada, H. Biosynthesis of the mycotoxin tenuazonic acid by a fungal NRPSPKS hybrid enzyme. Nat Commun 6, 8758 (2015).

42. Zhang, M.M. et al. CRISPR-Cas9 strategy for activation of silent Streptomyces biosynthetic gene clusters. Nat Chem Biol 13, 607-609 (2017).

43. Grubbs, K.J. et al. Large-scale bioinformatics analysis of Bacillus genomes uncovers conserved roles of natural products in bacterial physiology. mSystems 2, e00040-17 (2017).

44. Levy, R. \& Borenstein, E. Metabolic modeling of species interaction in the human microbiome elucidates community-level assembly rules. Proc Natl Acad Sci U S A 110, 12804-12809 (2013).

45. Griffin, A.S., West, S.A. \& Buckling, A. Cooperation and competition in pathogenic bacteria. Nature 430, 1024-1027 (2004).

46. Gu, S. et al. Competition for iron drives phytopathogen control by natural rhizosphere microbiomes. Nat Microbiol 5, 1002-1010 (2020).

47. Zhou, J. \& Ning, D. Stochastic community assembly: does it matter in microbial ecology? Microbiol Mol Biol R 81, e00002-17 (2017).

48. Landy, M., Rosenman, S.B. \& Warren, G.H. An antibiotic from Bacillus subtilis active against pathogenic fungi. J Bacteriol 54, 24 (1947).

49. Chaumeil, P.A., Mussig, A.J., Hugenholtz, P. \& Parks, D.H. GTDB-Tk: a toolkit to classify genomes with 
the Genome Taxonomy Database. Bioinformatics (2019).

50. Zhou, C. et al. pheS* , an effective host-genotype-independent counter-selectable marker for marker- 


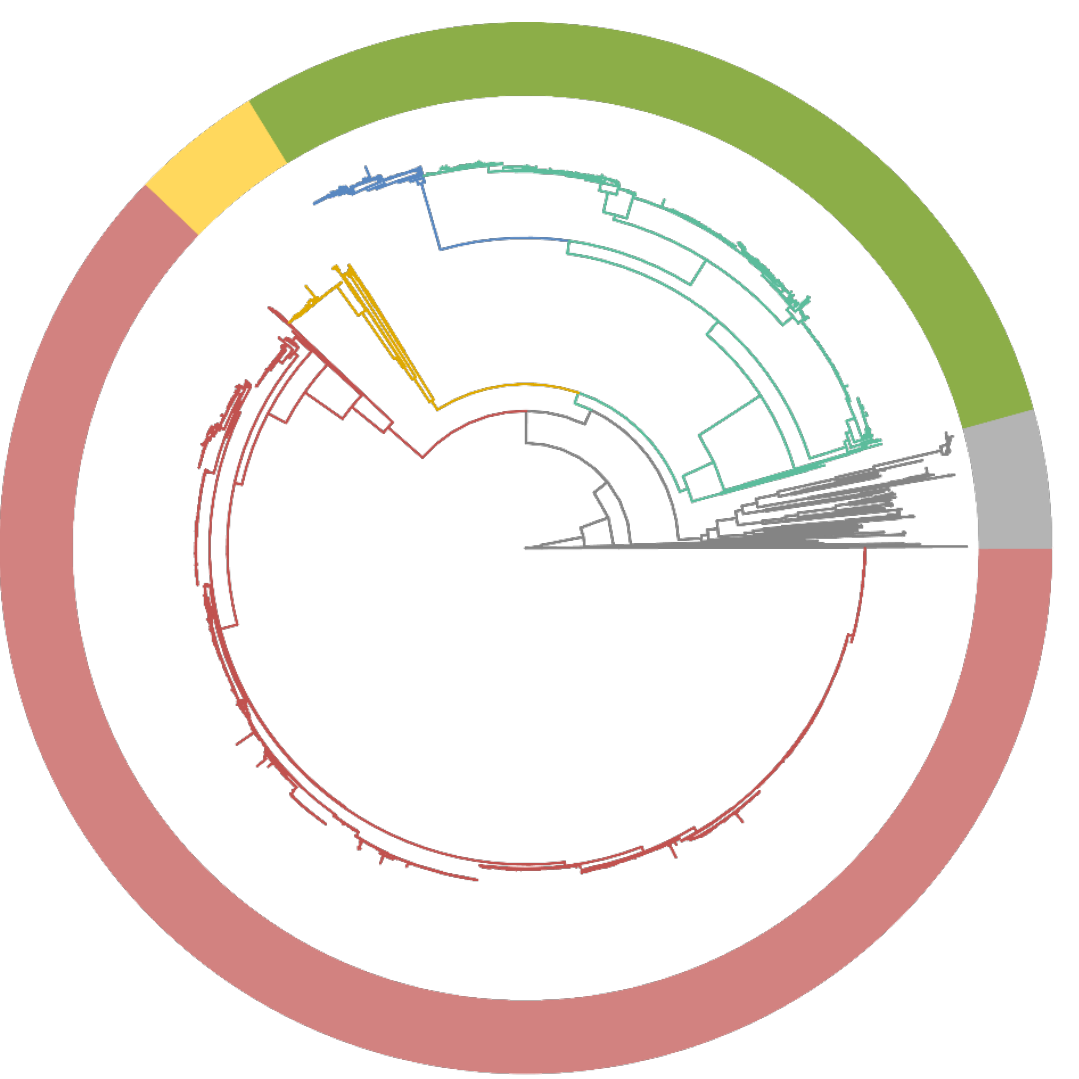

Clade

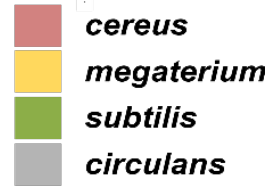

Clade / Subclade

- cereus

- megaterium

- subtilis / pumilus

_ subtilis / subtilis

- circulans

Fig. 1 Phylogram of the tested Bacillus genomes. The maximum likelihood (ML) phylogram of 4,268 Bacillus

genomes was based on the sequences of 120 ubiquitous single-copy proteins ${ }^{27}$. The phylogenetic tree shows

that Bacillus species can be generally clustered into the subtilis (light green circle; further includes subtilis 


\section{a}

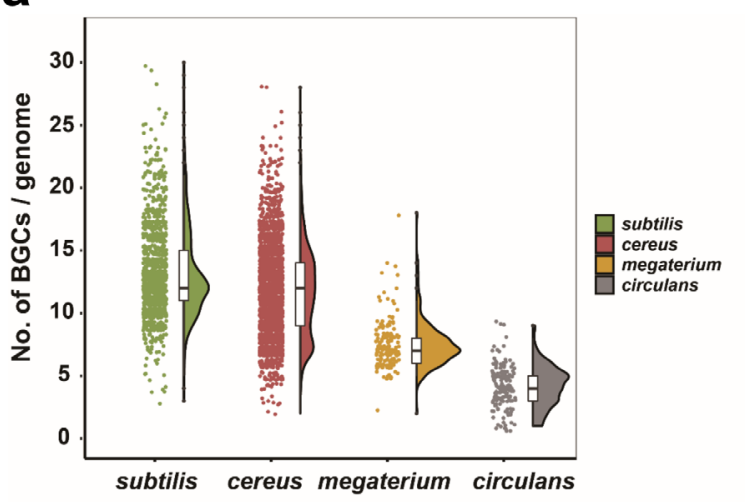

b

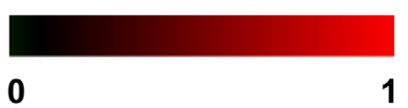

1

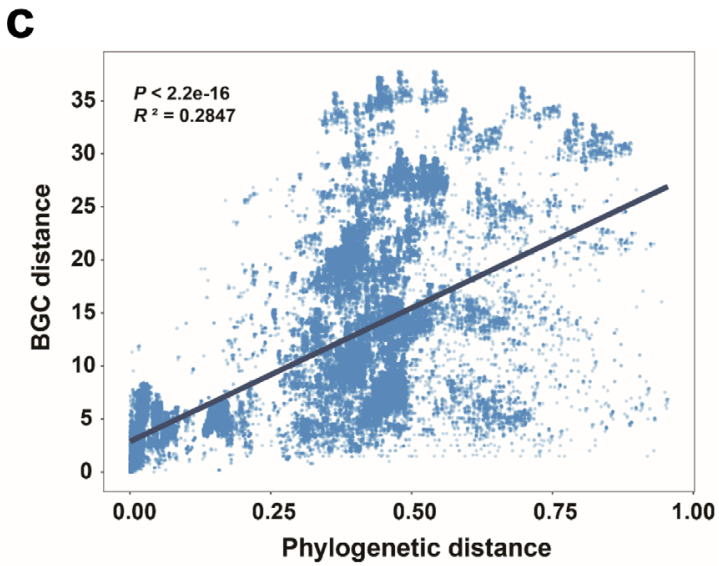

No. of BGCs from different families in each genome

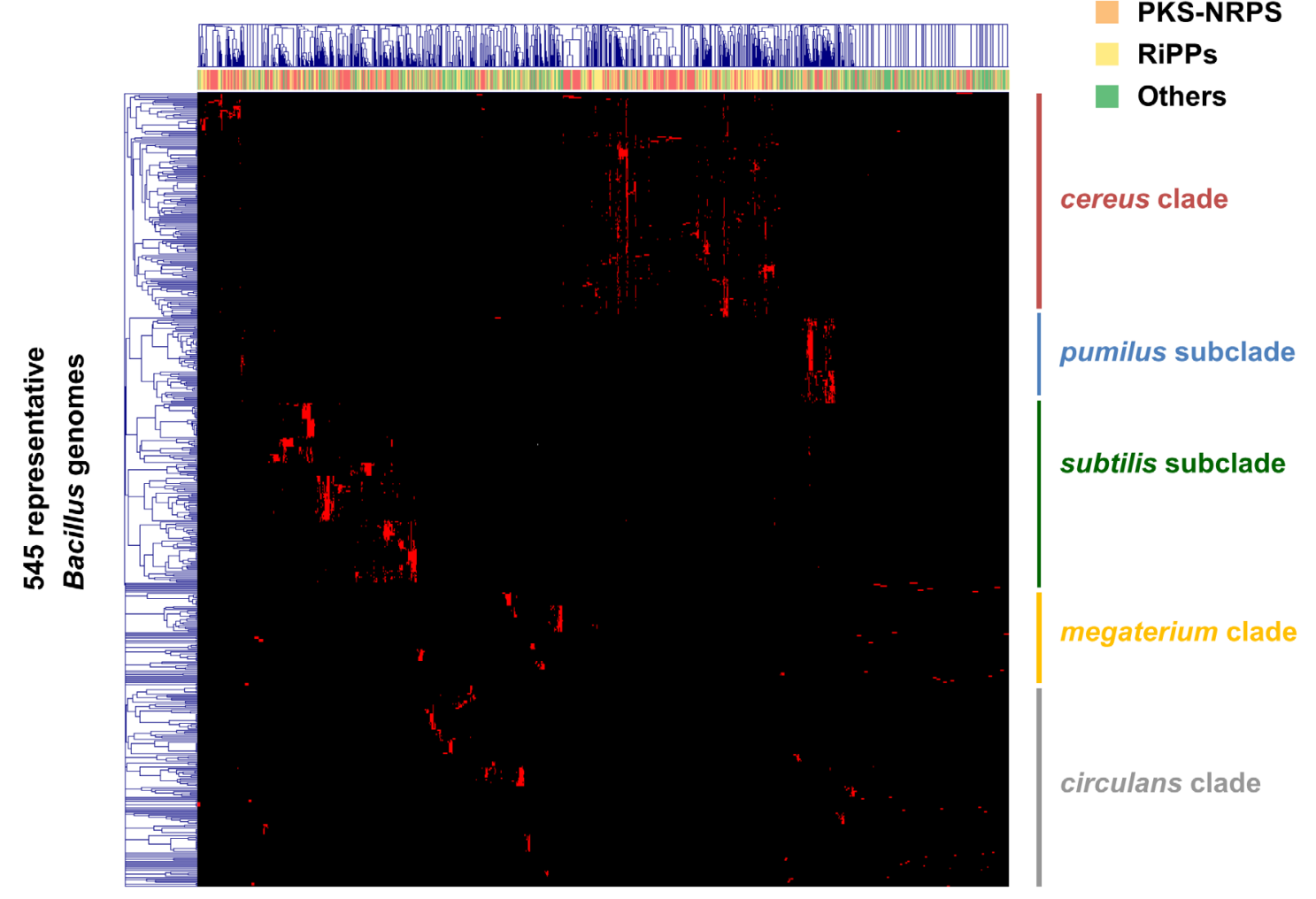

541 Fig. 2 Biosynthetic gene cluster (BGC) distribution is correlated with phylogeny in the genus Bacillus. (a) The 
543 Hierarchal clustering among the 545 representative Bacillus genomes based on the abundance of the different

544 biosynthesis gene cluster families (GCFs). Each row represents a GCF, which was classified through BiG-SCAPE

545 by calculating the Jaccard index (JI), adjacency index (AI), and domain sequence similarity (DSS) of each BGC ${ }^{28}$;

546 the color bar on the top of the heatmap represents the BGC class of each GCF, where PKS includes classes of

PKSother and PKSI, PKS-NRPS means PKS-NRPS Hybrids, Others includes classes of saccharides, terpene, and

others. Each line represents a Bacillus genome, and the abundance of each GCF in different genomes is shown

549 in the heatmap. The left tree was constructed based on the distribution pattern of GCFs, which showed a

550 similar pattern to the phylogram in Fig. 1. (c) The correlation between the BGC and phylogenetic distance of the 545 representative Bacillus genomes $\left(P<0.001, R^{2}=0.2847\right)$. 
a

\section{B. amyloliquefaciens ACCC19745}

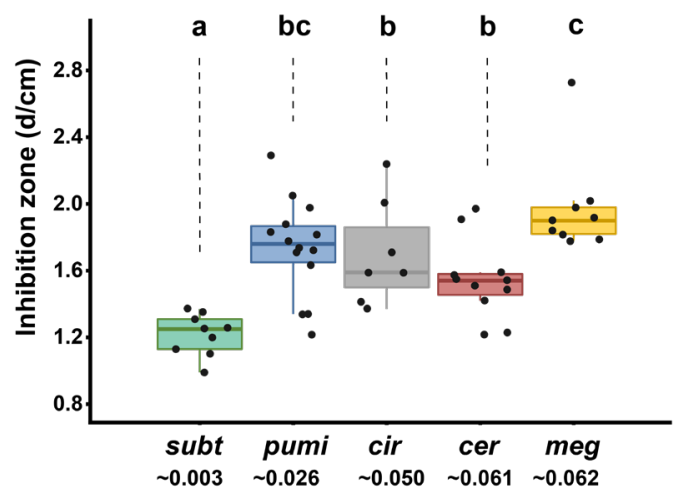

C

\section{B. thuringiensis YX7}

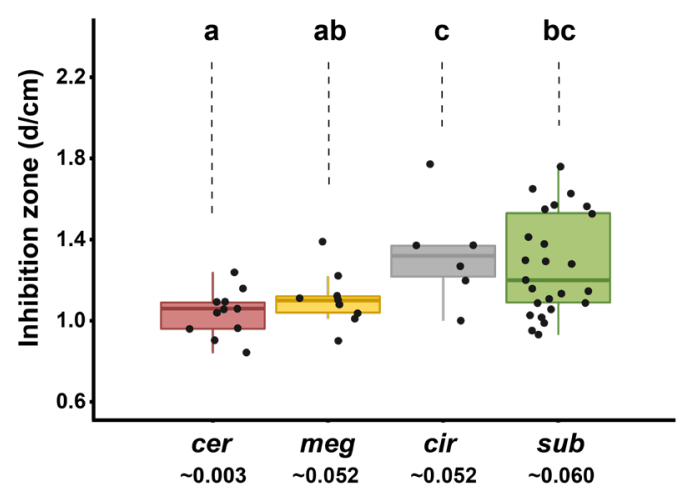

e

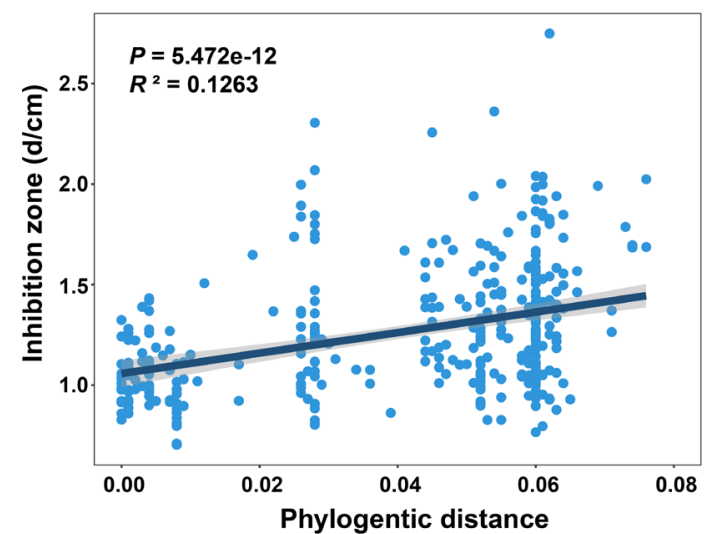

b

B. pumilus ACCC04450

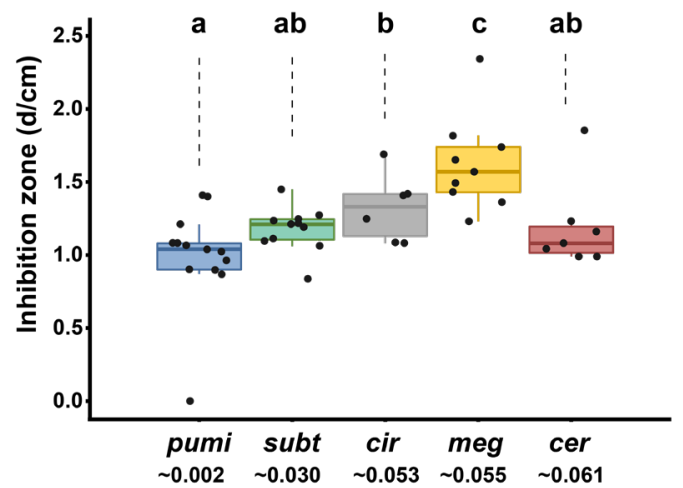

d

B. mobilis XL40

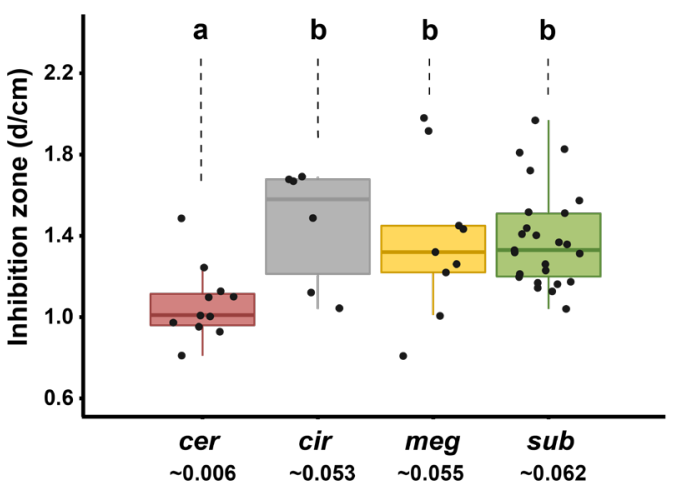

f

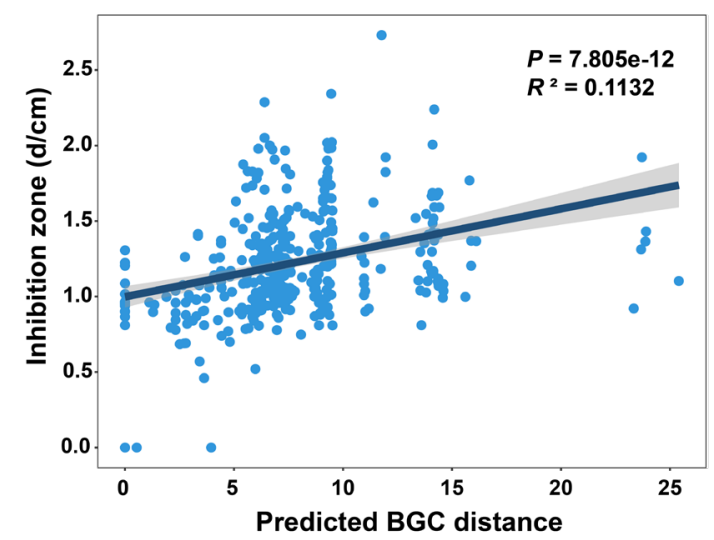

Fig. 3 Colony antagonism phenotype is positively correlated with the phylogenetic and BGC distance within 
Bacillus species. (a d) Inhibition of colonies of B. amyloliquefaciens ACCC19745, B. pumilus ACCC04450, B.

557 abbreviations indicates the average 16S rRNA gene phylogenetic distance of the target strains with the referred to the Bacillus genomes in the $\mathrm{NCBI}$ database that shared the highest $16 \mathrm{~S}$ rRNA similarity. 
bioRxiv preprint doi: https://doi.org/10.1101/2021.08 29.457868; this version posted August 30 2021. The copyright holder for this preprint

(which was not certified by peer review) is the author/funder, who has granted bioRxiv a license to display the preprint in perpetuity. It is made available under aCC-BY-NC-ND 4.0 International license.

a

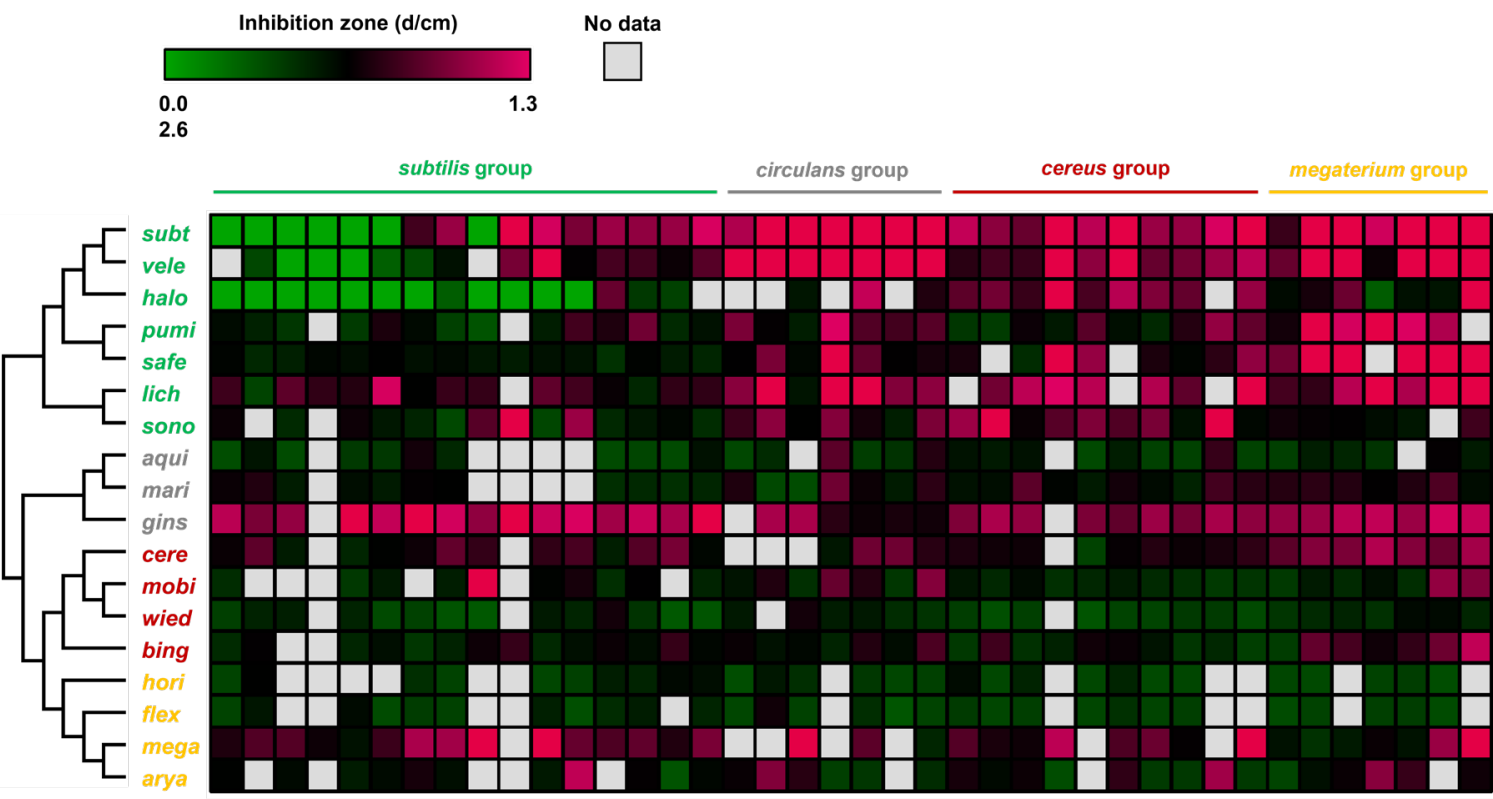

b

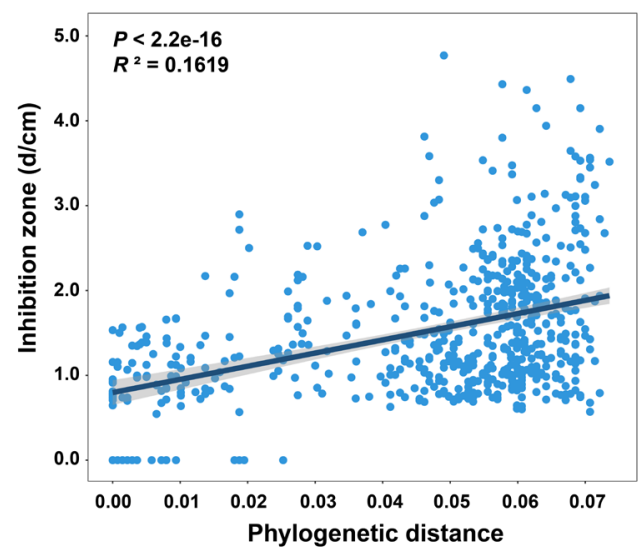

C

d

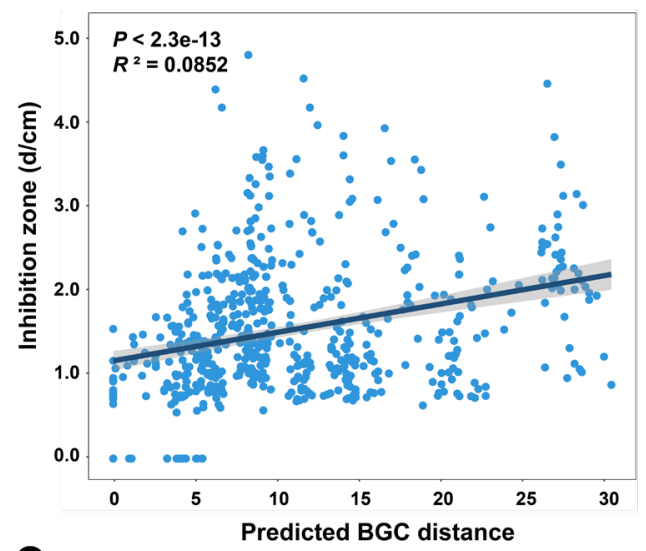

$\mathbf{e}$
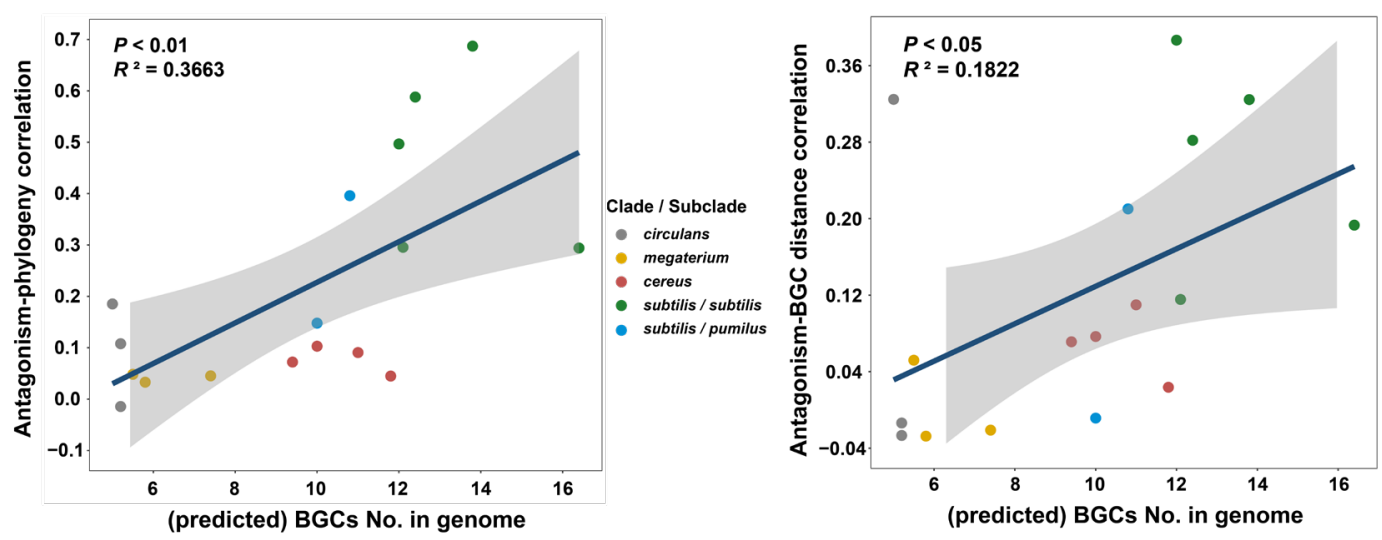
BGC distance in Bacillus. (a) Heatmap showing the antagonistic profiles of the fermentation supernatant of

$(\mathrm{ML})$ phylogenetic tree was constructed based on the 16S rRNA sequence of the 17 antagonistic strains: subt,

ACCC01509. Each inhibition assay includes three biological replicates and the average is shown in the heatmap

inhibition zone) and 16S rDNA phylogenetic distance (b) or the predicted BGC distance (c) among all the tested 


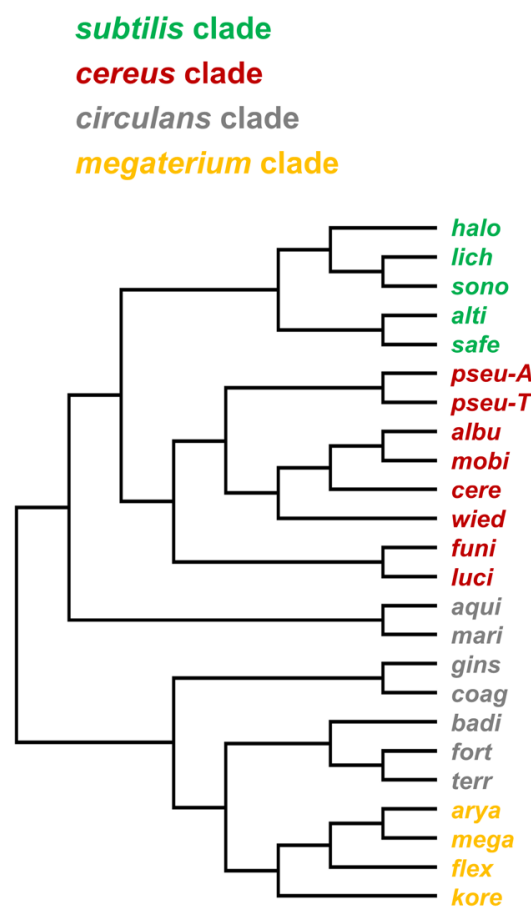

584
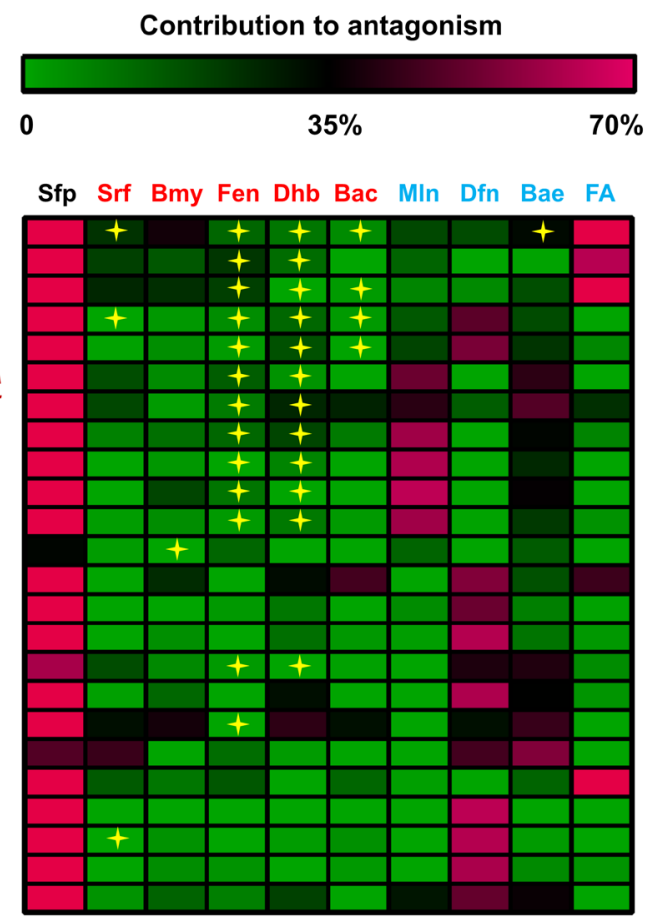

Antagonism (cm)

0.0

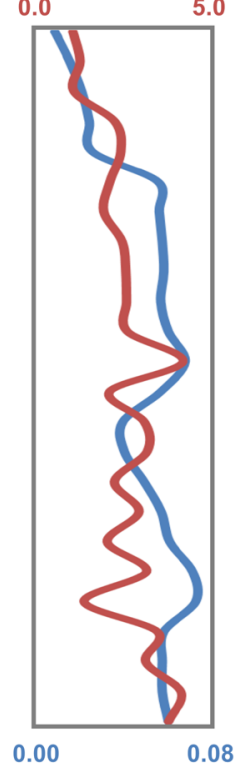

Phylogenetic distance

\section{Fig. 5 Contribution of BGCs to antagonizing Bacillus species from different clades by $B$. velezensis SQR9. The}

heatmap shows the contribution of each BGC product (on the top) to the inhibition of each target strain (on

the left), which was calculated as the percentage of the decreased inhibition zone of the corresponding BGC-

deficient mutant compared with wild type. The maximum likelihood (ML) tree on the left was constructed

based on the 16S rRNA sequences of the 24 target strains: halo, B. halotolerans CF7; lich, B. licheniformis LY2;

sono, B. sonorensis YX13; alti, B. altitudinis LY37; safe, B. safensis LY9; pseu-A, B. pseudomycoides ACCC10238;

pseu-B, B. pseudomycoides TZ8; albu, B. albus XL388; mobi, B. mobilis XL40; cere, B. cereus ACCC10263; wied,

B. wiedmannii CF23; funi, B. funiculus ACCC05674; luci, B. luciferensis XL165; aqui, B. aquimaris XL39; mari, B.

marisflavi XL37; gins, B. ginsengihumi ACCC05679; coag, B. coagulans ACCC10229; badi, B. badius ACCC60106;

fort, B. fortis ACCC10219; terr, B. terrae TL19; arya, B. aryabhattai XL26; mega, B. megaterium ACCC01509; 
Mln, macrolactin; Bae, bacillaene; Dfn, difficidin; FA, an antimicrobial fatty acid, bacillunoic acid. Specifically, 


\section{Supporting Information}

Table S1 Information of the 4,268 Bacillus genomes used for the phylogenetic analysis and biosynthetic gene cluster (BGC) prediction.

Table S2 Taxonomic distribution of genomes across different species and clades.

Table S3 Prediction of the putative biosynthetic gene clusters (BGCs) in all 4,268 Bacillus genomes by using antiSMASH.

Table S4 Statistics of different classes of biosynthetic gene clusters (BGCs) in different Bacillus clades.

Table S5 Biosynthesis gene cluster families (GCFs) and gene cluster clans (GCCs) in the 545 representative Bacillus genomes based on interactive sequence similarity network analysis using BiG-SCAPE.

Table S6 Product information of each predicted biosynthesis gene cluster family (GCF) and gene cluster clan (GCC) in the 545 representative Bacillus genomes.

Table S7 Statistics of biosynthetic gene clusters families (GCFs) in the $\mathbf{5 4 5}$ representative Bacillus genomes.

Table S8 Bacterial strains used in this study.

Table S9 Quantity of specific biosynthetic gene clusters (BGCs) of each antagonistic strain when confronted with different target strains in the fermentation supernatant inhibition assay. For strains whose genome have not been completely sequenced, the BGC presence was assigned if more than $80 \%$ of the corresponding Bacillus species genomes possessed this cluster, and their total predicted BGCs No. was calculated as the average BGCs. No. in all genomes of this species.

Table S10 Primers used for mutants construction and verification. 
of Fig. 1, which shows the detailed species information of the Bacillus genomes.

Fig. S2 Distribution of the biosynthetic gene clusters (BGCs) types. BGCs belonging to PKSI or EPS

630 are not shown in this figure since they are extremely rare in Bacillus genomes.

\section{Fig. S3 Profile of biosynthetic gene cluster (BGC) products and classification attached to the} phylogenetic tree of total 4,268 Bacillus genomes. Matching to the genome order in the phylogenetic tree (the same as that in Figs. 1 \& S1), BGCs profile in each genome were shown as the number of 256 detailed product types (Table S3) by the heatmap (which were further clustered only at the level of BGC products), as well as the number of 8 different classifications (NPRS, PKSI, PKSother, PKS-NRP_Hybrids, RiPPs, Saccharides, Terpene, and Others) through the histogram.

Fig. S4 Hierarchal clustering among the 545 representative Bacillus genomes based on the abundance of the different biosynthesis gene cluster families (GCFs). This figure is the fullyannotated version of Fig. 2 b. Each row represents a GCF and has been noted with the potential BGC product, which was classified through BiG-SCAPE by calculating the Jaccard index (JI), adjacency index (AI), and domain sequence similarity (DSS) of each $\mathrm{BGC}^{28}$. Each line represents a Bacillus genome, and the abundance of each GCF in different genomes is shown in the heatmap. The left tree was constructed based on the distribution pattern of BGCs from different families, which showed a similar pattern to the phylogram in Fig. 1.

Fig. S5 Connection of Bacillus genomes in 7 groups showing different correlation between biosynthetic gene clusters (BGCs) distance and phylogenetic distance. (a) The correlation between 
647 the BGC and phylogenetic distance of the 545 representative Bacillus genomes forms 7

648 distinguishable groups (group $1 \sim 7$ ). ( $\left.\mathbf{b}^{\sim} \mathbf{h}\right)$ For detail analysis, the points of 7 groups in (a),

649 corresponding to different relationships of two Bacillus genomes, were extracted, respectively, and

650 shown as the lines, which connected each two different genomes indicated by their own points. All

651 connected genomes were ranked as the orders in phylogenetic tree of the 545 Bacillus genomes

652 from top to bottom, as that shown in (b). Different colors were used to indicate each Bacillus

653 clades/subclades: red, cereus clade; blue, pumilus subclade; green, subtilis subclade; yellow,

654 megaterium clade; gray, circulans clade. Pd: phylogenetic distance; BGCd: BGC distance.

Fig. S6 Visual representations of colony confrontation and fermentation supernatant inhibition

assay.

Fig. S7 Correlation of the BGC-phylogenetic distance association with the (predicted) quantity of 